\title{
UNIFORM ERGODIC THEOREMS FOR LOCALLY INTEGRABLE SEMIGROUPS AND PSEUDO-RESOLVENTS
}

\author{
SEN - YEN SHAW \\ Dedicated to Professor Ky Fan
}

\begin{abstract}
We study uniform ergodicity (at $\infty$ ) of a locally integrable operator semigroup $T(\cdot)$ of type $w_{0}$ under a suitable condition which is weaker than the usual one " $w_{0} \leqslant 0$ ". We also give a precise characterization of the uniform Cesaro-ergodicity for semigroups of class $(0, A)$. To prove the part of Abel-ergodicity we first prove a general uniform ergodic theorem for pseudo-resolvents.
\end{abstract}

1. Introduction. Let $B(X)$ be the Banach algebra of all bounded linear operators on a Banach space $X$, and let $\{T(t) ; t>0\}$ be a family in $B(X)$ with the properties: (i) $T(s+t)=T(s) T(t)$ for all $s, t>0$; (ii) for each $x \in X$ the function $T(\cdot) x$ is Bochner integrable with respect to the Lebesgue measure over every finite subinterval of $(0, \infty)$. Such a family $T(\cdot)$ is called a locally integrable semigroup. It is known to be strongly continuous on $(0, \infty)$.

The type of $T(\cdot)$ is the number $w_{0}:=\inf _{t>0} t^{-1} \log \|T(t)\|<\infty$. For every $\lambda$ with $\operatorname{Re} \lambda>w_{0}$ and $x \in X$ the Bochner integral

$$
R(\lambda) x:=\int_{0}^{\infty} e^{-\lambda t} T(t) x d t
$$

exists and defines a bounded linear operator $R(\lambda)$ on $X$. The function $R(\lambda)$, $\operatorname{Re} \lambda>w_{0}$ (called the Laplace transform of $T(\cdot)$ ), satisfies the first resolvent equation

$$
R(\lambda)-R(\mu)=(\mu-\lambda) R(\lambda) R(\mu),
$$

that is, $R(\cdot)$ is a pseudo-resolvent on $\left\{\lambda \in \mathrm{C} ; \operatorname{Re} \lambda>w_{0}\right\}$ (cf. [2, p. 510]). $R(\cdot)$ has a unique maximal extension satisfying the above equation, and the domain of definition $\Omega$ of this maximally extended pseudo-resolvent, which we shall still denote by $R(\cdot)$, is an open subset of the complex plane $C$, on which $R(\cdot)$ is analytic and cannot be continued analytically beyond (cf. [2, pp. 188-189]). We denote $w:=\inf \{u \in(-\infty, \infty) ; \lambda \in \Omega$ for all $\lambda$ with $\operatorname{Re} \lambda>u\}$. Then $w \leqslant w_{0}$.

Received by the editors August 7, 1984 and, in revised form, August 29, 1985.

1980 Mathematics Subject Classification (1985 Revision). Primary 47A35, 47D05.

Key words and phrases. Cesàro-ergodicity, Abel-ergodicity, locally integrable semigroup, Laplace transform, pseudo-resolvent. 
One can also define the Laplace transform $R_{s}(\cdot)$ of $T(\cdot)$ in the following weaker sense:

$$
R_{s}(\lambda):=\text { so- } \lim _{t \rightarrow \infty} \int_{0}^{t} e^{-\lambda s} T(s) d s
$$

where the integral represents the operator which maps each $x \in X$ to the corresponding Bochner integral $\int_{0}^{t} e^{-\lambda s} T(s) x d s$. It can be proved (cf. [1, p. 248]) that if for a number $\lambda_{0} R_{s}\left(\lambda_{0}\right)$ exists, then $R_{s}(\lambda)$ exists for all $\lambda$ with $\operatorname{Re} \lambda>\operatorname{Re} \lambda_{0}$. Let us denote

$$
\begin{aligned}
\sigma & :=\inf \left\{u \in(-\infty, \infty) ; R_{s}(\lambda) \text { exists for all } \lambda \text { with } \operatorname{Re} \lambda>u\right\} \\
& =\inf \left\{u \in(-\infty, \infty) ; R_{s}(u) \text { exists }\right\}, \\
\sigma_{a} & :=\inf \left\{u \in(-\infty, \infty) ; R_{s}(u) \text { is analytic for all } \lambda \text { with } \operatorname{Re} \lambda>u\right\} .
\end{aligned}
$$

It is clear that $\sigma \leqslant \sigma_{a}, w \leqslant \sigma_{a} \leqslant w_{0}$, and that $R_{s}(\lambda)=R(\lambda)$ for all $\lambda$ with $\operatorname{Re} \lambda>w_{0}$, and hence, for all $\lambda$ with $\operatorname{Re} \lambda>\sigma_{a}$, by the uniqueness of analytic continuation.

It is known (cf. [1, Theorem 2.1]) that when $T(\cdot)$ is a semigroup of positive operators on an ordered Banach space, $R_{s}(\cdot)$ is analytic on $\{\lambda \in \mathbf{C} ; \operatorname{Re} \lambda>\sigma\}$, so that $w \leqslant \sigma_{a}=\sigma$. As will be seen in Proposition 7 , this actually holds for all semigroups of class $(0, A)$.

By a semigroup of class $(0, A)$ we mean that $T(\cdot)$ is locally integrable and $\lambda R(\lambda)$ converges strongly to $I$ as $\lambda \rightarrow \infty$. In this case, the operator $A^{0}: x \rightarrow$ $\lim _{t \rightarrow 0} t^{-1}(T(t)-I) x$ is densely defined and closable (cf. [2, pp. 342-344]). The closure $A$ of $A^{0}$ is called the infinitesimal generator of $T(\cdot) . T(\cdot)$ is of class $\left(C_{0}\right)$ if $T(t)$ converges strongly to $I$ as $t \rightarrow 0^{+}$. In this case, $T(\cdot)$ is also of class $(0, A)$ and $A^{0}$ is closed (see [2, p. 347]).

Let $S(t): x \rightarrow \int_{0}^{t} T(s) x d s(x \in X)$. The operators $C(t):=t^{-1} S(t), t>0$, are the Cesàro averages of $T(\cdot)$, and the operators $A(\lambda):=\lambda R(\lambda), \lambda>\sigma_{a}$, are the Abel averages of $T(\cdot)$. Uniform ergodic theorems are concerned with the uniform operator convergence of $C(t)$ as $t \rightarrow \infty$ and of $A(\lambda)$ as $\lambda \rightarrow 0^{+}$. The result of Hille and Phillips [2, Theorem 18.8.4] deals with the uniform Abel-ergodicity of semigroups of class $(A)$ (a class slightly larger than $(0, A)$ ) under the assumption " $w_{0} \leqslant 0$ ". The theorem of Lin [3] treats uniform Cesàro-ergodicity and Abelergodicity for semigroups of class $\left(C_{0}\right)$ under the assumption " $\lim _{t \rightarrow \infty}\|T(t)\| / t=0$ ", which also implies $w_{0} \leqslant 0$. However, as will be shown by an example in $\S 3$, it is possible for a semigroup to be uniformly ergodic while $-\infty=w=\sigma=\sigma_{a}<0<w_{0}$. Thus these well-known theorems do not apply universally.

The main purpose of this paper is to establish in $\$ 3$ a uniform ergodic theorem (Theorem 4) for a general locally integrable semigroup, assuming the weaker condition " $\sigma_{a} \leqslant 0$ ". Under this assumption, the condition " $\lim _{t \rightarrow \infty}\|T(t) R(1)\| / t=$ 0 " (but not $\lim _{t \rightarrow \infty}\|T(t)\| / t=0$ ) is necessary for uniform Cesàro-ergodicity of $T(\cdot)$. This is unlike the discrete case, where $\left\|T^{n}\right\| / n \rightarrow 0$ is necessary for the uniform ergodicity of $\left\{T^{n}\right\}$. When $T(\cdot)$ is a semigroup of class $(0, A)$, the uniform 
Cesàro-ergodicity can be characterized precisely (Theorem 6). We shall begin with a uniform ergodic theorem (Theorem 1) for a general pseudo-resolvent. It will apply in $\S 3$ to provide conditions for uniform Abel-ergodicity of $T(\cdot)$.

2. Uniform ergodic theorems for pseudo-resolvents. In this section $R(\cdot)$ is a general pseudo-resolvent on an open subset $\Omega$ of the complex plane $\mathbf{C}$.

THEOREM 1. Suppose that $0 \in \bar{\Omega}$. Then the following two statements are equivalent:

(1) There is a $P \in B(X)$ such that $\|\lambda R(\lambda)-P\| \rightarrow 0$ as $\lambda \rightarrow 0, \lambda \in \Omega$.

(2) $\left\|\lambda^{2} R(\lambda)\right\| \rightarrow 0$ as $\lambda \rightarrow 0$, and the range $\mathbf{R}(\lambda R(\lambda)-I)$ of $\lambda R(\lambda)-I$ is closed for some (and hence all) $\lambda \in \Omega$.

Proof. Note first that the range $\mathbf{R}(\lambda R(\lambda)-I)$ and the null space $\mathbf{N}(\lambda R(\lambda)-I)$ of $\lambda R(\lambda)-I$ are independent of $\lambda$ (see [6, p. 215]).

$(1) \Rightarrow(2)$. It is known from the mean ergodic theorem [6, p. 217] that $P$ is the projection onto $\mathbf{N}(\lambda R(\lambda)-I)$ along $\overline{\mathbf{R}(\lambda R(\lambda)-I)}$. The fact that

$$
\|\lambda R(\lambda)|\mathbf{N}(P)\|=\|(\lambda R(\lambda)-P)| \mathbf{N}(P)\| \leqslant\|\lambda R(\lambda)-P\| \rightarrow 0
$$

as $\lambda \rightarrow 0$ implies that $(\lambda R(\lambda)-I) \mid \mathbf{N}(P)$ is invertible for small $\lambda$ so that we have $\mathbf{R}(\lambda R(\lambda)-I) \supset \mathbf{N}(P)=\overline{\mathbf{R}(\lambda R(\lambda)-I)}$, i.e., $\mathbf{R}(\lambda R(\lambda)-I)$ is closed.

(2) $\Rightarrow(1)$. Fix a $\mu \neq 0$. Since $\mu R(\mu)-I$ has closed range, there exists a $M>0$ such that each $y$ in $\mathbf{R}(\mu R(\mu)-I)$ can be written as $y=(\mu R(\mu)-I) x$ for some $x$ satisfying $\|x\| \leqslant M\|y\|$. Using the resolvent equation we have

$$
\begin{aligned}
\|\lambda R(\lambda) y\| & =\|\lambda R(\lambda)(\mu R(\mu)-I) x\|=\left\|(\mu-\lambda)^{-1}\left[\lambda^{2} R(\lambda)-\lambda \mu R(\mu)\right] x\right\| \\
& \leqslant|\mu-\lambda|^{-1}\left[\left\|\lambda^{2} R(\lambda)\right\|+|\lambda|\|\mu R(\mu)\|\right] M\|y\|,
\end{aligned}
$$

which implies that $\|\lambda R(\lambda) \mid \mathbf{R}(\lambda R(\lambda)-I)\| \rightarrow 0$ as $\lambda \rightarrow 0$. Hence for small $\lambda$ the operator $K:=(\lambda R(\lambda)-I) \mid \mathbf{R}(\lambda R(\lambda)-I)$ is invertible and so we have that $\mathbf{R}(\lambda R(\lambda)-I)=\mathbf{R}(K)=\mathbf{R}\left((\lambda R(\lambda)-I)^{2}\right)$. From this one easily deduces that $X$ $=\mathbf{N}(\lambda R(\lambda)-I)+\mathbf{R}(\lambda R(\lambda)-I)$.

To show that the summation is direct, let $y$ be in $\mathbf{N}(\lambda R(\lambda)-I) \cap \mathbf{R}(\lambda R(\lambda)-I)$. Then $y=\lambda R(\lambda) y$ for all $\lambda \in \Omega$ and, as shown above, $\lambda R(\lambda) y \rightarrow 0$ as $\lambda \rightarrow 0$. Hence $y=0$ and so $X=\mathbf{N}(\lambda R(\lambda)-I) \oplus \mathbf{R}(\lambda R(\lambda)-I)$. Let $P$ be the projection onto $\mathbf{N}(\lambda R(\lambda)-I)$ along $\mathbf{R}(\lambda R(\lambda)-I)$. Clearly we have $\|\lambda R(\lambda)-P\|=\| 0 \oplus$ $[\lambda R(\lambda) \mid \mathbf{R}(\lambda R(\lambda)-I)] \| \rightarrow 0$ as $\lambda \rightarrow 0$.

Corollary 2. Suppose that the domain $\Omega$ of $R(\cdot)$ is unbounded. Then the following statements are equivalent:

(1) There is a $Q \in B(X)$ such that $\|\lambda R(\lambda)-Q\| \rightarrow 0$ as $|\lambda| \rightarrow \infty, \lambda \in \Omega$.

(2) $\|R(\lambda)\| \rightarrow 0$ as $|\lambda| \rightarrow \infty$, and $\mathbf{R}(R(\lambda)$ ) is closed for some (and hence all) $\lambda \in \Omega$.

(3) $R(\lambda)=Q(\lambda I-A)^{-1}$ for some $Q, A \in B(X)$ satisfying $Q^{2}=Q, A Q=Q A$ $=A$.

Proof. Define $R_{1}(\lambda):=1 / \lambda-\left(1 / \lambda^{2}\right) R(1 / \lambda)$ for $\lambda \in \Omega_{1}:=\{\lambda \in C ; 1 / \lambda \in \Omega\}$. An easy computation shows that $R_{1}(\cdot)$ is a pseudo-resolvent on $\Omega_{1}$, which has the limit point 0 . Therefore, Theorem 1 applies to $R_{1}(\cdot)$, and the equivalence of (1) and 
(2) follows by the facts that $\left\|\lambda^{2} R_{1}(\lambda)\right\| \rightarrow 0$ as $\lambda \rightarrow 0, \lambda \in \Omega_{1}$, if and only if $\|R(\lambda)\| \rightarrow 0$ as $|\lambda| \rightarrow \infty, \lambda \in \Omega$, and that $\left\|\lambda R_{1}(\lambda)-P\right\| \rightarrow 0$ as $\lambda \rightarrow 0$ if and only if $\|\lambda R(\lambda)-Q\| \rightarrow 0$ as $|\lambda| \rightarrow \infty$, where $Q=I-P$. “(3) $\Rightarrow(1)$ " is obvious, and “(1) $\Rightarrow(3)$ " is proved in Theorem 18.8.2 of [2].

REMARK. The operators $P$ and $Q$ turn out to be the linear projections with $\mathbf{R}(P)=\mathbf{N}(\lambda R(\lambda)-I), \quad \mathbf{N}(P)=\mathbf{R}(\lambda R(\lambda)-I), \quad \mathbf{R}(Q)=\mathbf{R}(R(\lambda))$ and $\mathbf{N}(Q)=$ $\mathbf{N}(R(\lambda))$ for all $\lambda \in \Omega$.

3. Uniform ergodic theorems for locally integrable semigroups. All well-known uniform ergodic theorems for semigroups have been formulated for those of type $w_{0} \leqslant 0$. We shall first give an example of a uniformly ergodic semigroup of class $\left(C_{0}\right)$ which satisfies $-\infty=\sigma_{a}<0<w_{0}$, and then prove a uniform ergodic theorem for general locally integrable semigroups under the assumption $\sigma_{a} \leqslant 0$.

EXAMPLE. Let $1 \leqslant p<q<\infty$, and let $X$ be the set of all Lebesgue measurable functions $f$ on $(0, \infty)$ such that

$$
\|f\|:=\left(\int_{0}^{\infty} e^{p s^{2}}|f(s)|^{p} d s\right)^{1 / p}+\left(\int_{0}^{\infty}|f(s)|^{q} d s\right)^{1 / q}<\infty
$$

Then $(X,\|\cdot\|)$ is a Banach lattice which is reflexive whenever $p>1$. For $\alpha \geqslant 0$ let $T_{\alpha}(\cdot)$ be the semigroup defined by $\left(T_{\alpha}(t) f\right)(s):=e^{\alpha t} f(t+s)(f \in X, s, t \geqslant 0)$. Then $T_{\alpha}(t)=e^{\alpha(t)} T_{0}(t)$.

It was shown in [1] that $\left\|T_{0}(t)\right\|=1$ for all $t \geqslant 0$ and for $T_{0}(\cdot) w=\sigma_{a}=\sigma=-\infty$. Hence $T_{\alpha}(\cdot)$ has type $w_{0}=\alpha$ and $w=\sigma_{a}=\sigma=-\infty$ for all $\alpha \geqslant 0$. Thus the infinitesimal generator $A_{\alpha}=d / d s+\alpha I$ of $T_{\alpha}(\cdot)$ has empty spectrum, and so

$$
\|A(\lambda)\|=\left\|\lambda\left(\lambda I-A_{\alpha}\right)^{-1}\right\| \rightarrow 0 \cdot\left\|A_{\alpha}^{-1}\right\|=0 \quad \text { as } \lambda \rightarrow 0 .
$$

Since $T_{\alpha}(t)$ are positive operators, we have for any nonnegative function $f$ in $X$

$$
\begin{aligned}
C(t) f & =t^{-1} \int_{0}^{t} T_{\alpha}(s) f d s \leqslant t^{-1} \int_{0}^{t} e^{1-s / t} T_{\alpha}(s) f d s \\
& \leqslant t^{-1} e \int_{0}^{\infty} e^{-s / t} T_{\alpha}(s) f d s=e t^{-1} R\left(t^{-1}\right) f=e A\left(t^{-1}\right) f .
\end{aligned}
$$

It follows that $\|C(t)\| \leqslant e\left\|A\left(t^{-1}\right)\right\| \rightarrow 0$ as $t \rightarrow \infty$ (cf. [4, pp. 81 and 230]). Hence $T_{\alpha}(\cdot)$ is uniformly Abel and Cesàro ergodic to 0 .

We shall need the following lemma in the proof of Theorem 4:

LEMMA 3. Let $T(\cdot)$ be a locally integrable semigroup, and let $S(t)$ and $R_{s}(\lambda)$ be as defined in $\S 1$. The equality $(T(t)-I) R_{s}(\lambda)=S(t)\left(\lambda R_{s}(\lambda)-I\right)$ holds for all $t>0$ and $\operatorname{Re} \lambda>\sigma$.

Proof. Integration by parts gives that

$$
R_{s}(\lambda) x=\int_{0}^{\infty} e^{-\lambda u} T(u) x d u=\lambda \int_{0}^{\infty} e^{-\lambda u} S(u) x d u .
$$

Then we use the identity $(T(t)-I) S(u)=S(t)(T(u)-I)$ (see [5]) to obtain that

$$
\begin{aligned}
(T(t)-I) R_{s}(\lambda) x & =\lambda \int_{0}^{\infty} e^{-\lambda u}(T(t)-I) S(u) x d u \\
& =\lambda \int_{0}^{\infty} e^{-\lambda u} S(t)(T(u)-I) x d u=S(t)\left(\lambda R_{s}(\lambda)-I\right) x .
\end{aligned}
$$


TheORem 4. Let $T(\cdot)$ be a locally integrable semigroup. Assume that $\sigma_{a} \leqslant 0$. Then the following statements are equivalent:

(1) $T(\cdot)$ is uniformly Cesàro-ergodic.

(2) $\|T(t) R(1)\| / t \rightarrow 0$ as $t \rightarrow \infty$, and $T(\cdot)$ is uniformly Abel-ergodic.

(3) $\|T(t) R(1)\| / t \rightarrow 0$ as $t \rightarrow \infty$, and $\mathbf{R}(R(1)-I)$ is closed.

Proof. (1) $\Rightarrow(2)$. We have for each $x \in X$ and $a>0$,

$$
\begin{aligned}
\|(A(\lambda)-P) x\| & =\left\|\lambda^{2} \int_{0}^{\infty} e^{-\lambda t}(S(t)-t P) x d t\right\| \\
& \leqslant\left[\lambda^{2} \int_{0}^{a} e^{-\lambda t}(\|S(t)\|+t\|P\|) d t+\lambda^{2} \int_{a}^{\infty} e^{-\lambda t} t\|C(t)-P\| d t\right]\|x\| \\
& \leqslant\left[\left(\sup _{0 \leqslant t \leqslant a}\|S(t)\|+a\|P\|\right) \lambda^{2} a+\sup _{t>a}\|C(t)-P\|\right]\|x\| .
\end{aligned}
$$

If $\|C(t)-P\| \rightarrow 0$ as $t \rightarrow \infty$, then it is easy to see from the above estimate that $\|A(\lambda)-P\| \rightarrow 0$ as $\lambda \rightarrow 0^{+}$. Then the fact that $\mathbf{N}(P)=\mathbf{R}(R(1)-I)$ (see the remark in §2) and Lemma 3 imply

$$
\begin{aligned}
\|(T(t)-I) R(1)\| / t & =\|C(t)(R(1)-I)\|=\|(C(t)-P)(R(1)-I)\| \\
& \leqslant\|C(t)-P\|\|R(1)-I\| \rightarrow 0 \quad(t \rightarrow \infty) .
\end{aligned}
$$

Hence the statement (2) holds when (1) holds.

" $(2) \Rightarrow(3)$ " is contained in Theorem 1.

(3) $\Rightarrow(1)$. First we prove that $\lim _{t \rightarrow \infty}\|T(t) R(1)\| / t=0$ implies $\lim _{\lambda \rightarrow 0^{+}}\left\|\lambda^{2} R(\lambda)\right\|=0$. Given $\varepsilon>0$, let $a>0$ be such that $\|T(t) R(1)\| \leqslant \varepsilon t$ for all $t>a$. Using the resolvent equation we have for every $x \in X$

$$
\begin{aligned}
\left\|\lambda^{2} R(\lambda) x\right\| & =\left\|\lambda^{2}[R(1)+(1-\lambda) R(\lambda) R(1)] x\right\| \\
& \leqslant \lambda^{2}\|R(1)\|\|x\|+|1-\lambda| \lambda^{2} \int_{0}^{\infty} e^{-\lambda t}\|T(t) R(1) x\| d t \\
& \leqslant \lambda^{2}\|R(1)\|\|x\|+|1-\lambda|\left[\lambda^{2} \int_{0}^{a}\|T(t) R(1) x\| d t+\varepsilon \lambda^{2} \int_{a}^{\infty} e^{-\lambda t} t d t\|x\|\right] \\
& \leqslant\left\{\lambda^{2}\|R(1)\|+|1-\lambda|\left[\lambda^{2}\|W(a)\|\|R(1)\|+\varepsilon\right]\right\}\|x\|,
\end{aligned}
$$

where $W(a)$ denotes the operator from $X$ to $L_{1}(X,[0, a])$ defined by $W(a) x=$ $T(\cdot) x$, which is known to be bounded (cf. [2, p. 58]). It is easily seen from the above estimate that $\left\|\lambda^{2} R(\lambda)\right\| \rightarrow 0$ as $\lambda \rightarrow 0^{+}$.

Now the statement (2) of Theorem 1 holds, and it was proved there that

$$
X=\mathbf{N}(R(1)-I) \oplus \mathbf{R}(R(1)-I) .
$$

Let $K$ be the restriction of $R(1)-I$ to $\mathbf{R}(R(1)-I)$. Then $K$ is one-to-one, onto, and hence invertible. For $x \in \mathbf{R}(R(1)-I)$ let $y=K^{-1} x$. By Lemma 3 we have

$$
\begin{aligned}
\|C(t) x\| & =\|C(t)(R(1)-I) y\|=\left\|t^{-1}(T(t)-I) R(1) K^{-1} x\right\| \\
& \leqslant t^{-1}(\|T(t) R(1)\|+\|R(1)\|)\left\|K^{-1}\right\|\|x\| .
\end{aligned}
$$


This shows that $\|C(t) \mid \mathbf{N}(R(1)-I)\| \rightarrow 0$ as $t \rightarrow \infty$. Let $P$ be the projection onto $\mathbf{R}(R(1)-I)$ along $\mathbf{R}(R(1)-I)$. In order to prove that $\|C(t)-P\|$ tends to 0 as $t \rightarrow \infty$, it remains to show that the restriction of $C(t)$ to $\mathbf{N}(R(1)-I)$ is an identity map for all $t>0$. It suffices to show that $\mathbf{N}(R(1)-I) \subset \mathbf{N}(T(t)-I)$. Let $x \in$ $\mathbf{N}(R(1)-I)$. Then $x \in \mathbf{N}(\lambda R(\lambda)-I)$ for all $\lambda>\sigma_{a}[6$, p. 215], so that

$$
\begin{aligned}
(T(t)-I) x & =\lambda(T(t)-I) R(\lambda) x \\
& =\lambda S(t)(\lambda R(\lambda)-I) x=S(t)\left(\lambda^{2} R(\lambda)-\lambda\right) x,
\end{aligned}
$$

which converges to 0 as $\lambda \rightarrow 0^{+}$. Hence $x$ belongs to $\mathbf{N}(T(t)-I)$. The proof is now completed.

COROLlaRY 5. Let $T(\cdot)$ be a locally integrable semigroup satisfying $\sigma_{a} \leqslant 0$ and $\|T(t) R(1)\| / t \rightarrow 0(t \rightarrow \infty)$. Then the following statements are equivalent:

(1) $T(\cdot)$ is uniformly Cesàro-ergodic.

(2) $T(\cdot)$ is uniformly Abel-ergodic.

(3) $\mathbf{R}(R(1)-I)$ is closed.

Remarks. (1) If $T(\cdot)$ is of class $(0, A)$ with generator $A$, one has that $\mathbf{R}(R(1)-I)$ $=\mathbf{R}\left((I-A)^{-1}-I\right)=\mathbf{R}\left(A(I-A)^{-1}\right)=\mathbf{R}(A)$. Thus the theorem of Lin [3] is a specialization of Corollary 5.

(2) It follows from Theorem 4 that the semigroup $T_{\alpha}(\cdot)$ in the previous example satisfies $\left\|T_{\alpha}(t) R(1)\right\| / t \rightarrow 0(t \rightarrow \infty)$, while $\left\|T_{\alpha}(t)\right\| / t=e^{\alpha t} t \rightarrow \infty$ in case $\alpha>0$. Therefore, the hypothesis in Corollary 5 is in general strictly weaker than Lin's $(\|T(t)\| / t \rightarrow 0)$, and it cannot be further weakened.

The next theorem gives a precise characterization for the uniform Cesàro-ergodicity of $(0, A)$ semigroups.

THEOREM 6. Let $T(\cdot)$ be a semigroup of class $(0, A)$. Then $T(\cdot)$ is uniformly Cesàro ergodic if and only if (i) $\sigma \leqslant 0$, (ii) $\mathbf{R}(A)$ is closed, and (iii) $\|T(t) R(1)\| / t \rightarrow 0$ as $t \rightarrow \infty$.

This theorem is deduced from Theorem 4 and the following two propositions.

Proposition 7. If $T(\cdot)$ is a semigroup of class $(0, A)$, then $\sigma=\sigma_{a}$.

Proof. Since $\sigma \leqslant \sigma_{a}$, it suffices to show that if $\operatorname{Re} \lambda>\sigma$, then $\lambda I-A$ is invertible and $R_{s}(\lambda)=(\lambda I-A)^{-1}$.

We first prove that $\left(\lambda I-A^{0}\right) R_{s}(\lambda) x=x$ for $x \in D\left(A^{0}\right)$. Let

$$
A_{h}=h^{-1}(T(h)-I) \text {. }
$$

Then we have

$$
\begin{aligned}
A_{h} R_{s}(\lambda) x & =A_{h} \lim _{t \rightarrow \infty} \int_{0}^{t} e^{-\lambda s} T(s) x d s \\
& =h^{-1} \lim _{t \rightarrow \infty} \int_{0}^{t} e^{-\lambda s}[T(s+h) x-T(s) x] d s \\
& =h^{-1}\left(e^{\lambda h}-1\right) R_{s}(\lambda) x-h^{-1} \int_{0}^{h} e^{\lambda(h-s)} T(s) x d s,
\end{aligned}
$$


which tends to $\lambda R_{s}(\lambda) x-x$ as $h \rightarrow 0$ if $x \in D\left(A^{0}\right)$, by the continuity of $T(t) x$ at $t=0$. Hence $R_{s}(\lambda) x \in D\left(A^{0}\right)$ and $A^{0} R_{s}(\lambda) x=\lambda R_{s}(\lambda) x-x$.

If $y \in X$, we can obtain a sequence $y_{n} \in D\left(A^{0}\right)$ such that $y_{n} \rightarrow y$. Then we have that $R_{s}(\lambda) y_{n} \rightarrow R_{s}(\lambda) y$ and $A^{0} R_{s}(\lambda) y_{n}-y_{n}=\lambda R_{s}(\lambda) y_{n}-y_{n} \rightarrow \lambda R_{s}(\lambda) y-y$. Since $A$ is the closure of $A^{0}$, we have proved that $R_{s}(\lambda) X \subset D(A)$ and $(\lambda I-A) R_{s}(\lambda)=I$.

It remains to show that $A R_{s}(\lambda) x=R_{s}(\lambda) A x$ for $x \in D(A)$. Given $x \in D(A)$ there exists a sequence $x_{n} \in D\left(A^{0}\right)$ such that $x_{n} \rightarrow x$ and $A^{0} x_{n} \rightarrow A x$. Since $A_{h} R_{s}(\lambda) x_{n}=R_{s}(\lambda) A_{h} x_{n}$, by letting $h \rightarrow 0$ we obtain that $A^{0} R_{s}(\lambda) x_{n}=$ $R_{s}(\lambda) A^{0} x_{n}$. Therefore, $A^{0} R_{s}(\lambda) x_{n}$ tends to $R_{s}(\lambda) A x$ as $n \rightarrow \infty$. This and the fact that $R_{s}(\lambda) x_{n} \rightarrow R_{s}(\lambda) x$ show that $R_{s}(\lambda) x \in D(A)$ and $A R_{s}(\lambda) x=R_{s}(\lambda) A x$.

PROPOSITION 8. If a locally integrable semigroup $T(\cdot)$ is strongly Cesàro-ergodic, then $\sigma \leqslant 0$, i.e. the Laplace transform $R_{s}(\lambda)$ exists for all $\lambda$ with $\operatorname{Re} \lambda>0$.

Proof. The uniform boundedness principle implies that $\|C(t)\| \leqslant M$ for all $t>1$. Let $\operatorname{Re} \lambda>0$. We have for all $v>u>0$ and $x \in X$

$$
\begin{aligned}
\left\|\int_{u}^{v} e^{-\lambda t} T(t) x d t\right\| & =\left\|\left.e^{-\lambda t} S(t) x\right|_{u} ^{v}+\lambda \int_{u}^{v} e^{-\lambda t} S(t) x d t\right\| \\
& \leqslant\left\{\left|e^{-\lambda v}\right| v+\left|e^{-\lambda u}\right| u+|\lambda| \int_{u}^{v} e^{-t \operatorname{Re} \lambda} t d t\right\} M\|x\|,
\end{aligned}
$$

which shows that $\left\|\int_{u}^{v} e^{-\lambda t} T(t) d t\right\| \rightarrow 0$ as $u \rightarrow \infty$. Hence

$$
R_{s}(\lambda)=\text { uo- } \lim _{t \rightarrow \infty} \int_{0}^{u} e^{-\lambda t} T(t) d t
$$

exists.

ACKNOWLEDGMENT. The author wishes to thank the referee for his valuable suggestion.

\section{REFERENCES}

1. G. Greiner, J. Voigt, and M. Wolff, On the spectral bound of the generator of semigroups of positive operators, J. Operator Theory 5 (1981), 245-256.

2. E. Hille and R. S. Phillips, Functional analysis and semigroups, Amer. Math. Soc. Colloq. Publ., vol. 31, Amer. Math. Soc., Providence, R. I., 1957.

3. M. Lin, On the uniform ergodic theorem. II, Proc. Amer. Math. Soc. 46 (1974), 217-225.

4. H. H. Schaefer, Banach lattices and positive operators, Springer-Verlag, Berlin-Heidelberg-New York, 1974.

5. S.-Y. Shaw, Ergodic properties of operator semigroups in general weak topologies, J. Funct. Anal. 49 (1982), 152-169.

6. K. Yosida, Functional analysis, 3rd ed., Springer-Verlag, New York, 1971.

Department of Mathematics, National Central University, Chung - Li, Taiwan 320, Republic OF CHINA 